\title{
The effect of lipoic acid in the prevention of myocardial infarction in diabetic rats
}

\author{
Ozgun $E^{1}$, Sayilan Ozgun $\mathrm{G}^{1}$, Usta $\mathrm{U}^{2}$, Eskiocak $\mathrm{S}^{1}$, Sut $\mathrm{N}^{3}$, Suer Gokmen $\mathrm{S}^{1}$ \\ Department of Medical Biochemistry, Trakya University School of Medicine, Edirne, Turkey. \\ drozgune@hotmail.com
}

\begin{abstract}
OBJECTIVES: We aimed to investigate the effect of lipoic acid in the prevention of myocardial infarction in diabetic rats.

METHODS: Rats were divided into five groups as control, ISO, LA+ISO, STZ+ISO and STZ+LA+ISO. To induce diabetes, single dose of streptozotocin was injected to STZ+ISO and STZ+LA+ISO groups. Lipoic acid (10 mg/ $\mathrm{kg} /$ day) was injected for 14 days to $L A+I S O$ and STZ+LA+ISO groups. To induce myocardial infarction, isoproterenol was injected to ISO, LA+ISO, STZ+ISO and STZ+LA+ISO groups on the days 13 and 14 of lipoic acid treatment. Cardiac necrosis and leucocyte infiltration were investigated histopathologically. Serum malondialdehyde levels, paraoxonase and lactonase activities were measured spectrophotometrically.

RESULTS: Isoproterenol caused a significant increase in cardiac necrosis, leucocyte infiltration and serum lipid peroxidation whereas a significant decrease in serum paraoxonase and lactonase activities. In myocardial infarcted non-diabetic rats, while lipoic acid caused a significant decrease in cardiac necrosis, leucocyte infiltration and serum lipid peroxidation and a significant increase in serum paraoxonase and lactonase activities, it did not change these histopathologic or biochemical parameters in myocardial infarcted diabetic rats.

CONCLUSION: Lipoic acid, at the dose of $10 \mathrm{mg} / \mathrm{kg}$, is effective to prevent myocardial infarction in non-diabetic rats but it is insufficient in diabetic rats (Tab. 1, Fig. 2, Ref. 35).

KEY WORDS: streptozotocin-induced diabetes, lipoic acid, isoproterenol-induced myocardial infarction, lipid peroxidation, paraoxonase, lactonase.
\end{abstract}

\section{Introduction}

Myocardial infarction can be defined as myocardial cell death due to a prolonged ischemia and it is a major cause of death and disability worldwide (1). Oxidative stress is increased in patients with myocardial infarction (2). High doses of isoproterenol (ISO) induce experimental myocardial infarction which has similar histopathological changes in cardiac tissue when compared with cardiac tissue of the patients with myocardial infarction $(3,4)$. Oxidative stress plays an important role also in the pathogenesis of ISO-induced myocardial infarction $(5,6)$.

Diabetes mellitus (DM) is a metabolic derangement with hyperglycemia and it's a global health problem. DM has several microvascular and macrovascular complications, which reduce health-related quality of life and increase mortality (7). DM is a risk factor for myocardial infarction (8) and oxidative stress is one of the primary causes of myocardial infarction in diabetics (9). Streptozotocin (STZ) is a frequently used chemical to induce experimental diabetes with its cytotoxic effect on pancreatic beta cells (10).

${ }^{1}$ Department of Medical Biochemistry, Trakya University School of Medicine, Edirne, Turkey, ${ }^{2}$ Department of Pathology, Trakya University School of Medicine, Edirne, Turkey, and ${ }^{3}$ Department of Biostatistics and Medical Informatics, Trakya University School of Medicine, Edirne, Turkey

Address for correspondence: E. Ozgun, Department of Medical Biochemistry, Trakya University School of Medicine 22030 Edirne, Turkey. Phone: +905444191946, Fax: +902842357652
Hyperglycemia increases oxidative stress, which causes damages to biological molecules, such as protein, lipid and DNA and oxidative stress is associated with complication of DM $(9,11)$. Malondialdehyde (MDA) is the end product and a useful marker of lipid peroxidation (12).

Paraoxonase (PON) enzyme family has three members including PON1, PON2 and PON3, which are primarily synthesized by the liver. PON1 and PON3 are antioxidant enzymes bound to HDL in circulation and have anti-atherogenic properties (13). PON enzymes have different activities toward different substrates, but their primary activity is lactanose activity (14). It has been shown that serum PON enzyme activities are decreased in both myocardial infarction $(15,16)$ and diabetes $(17,18)$.

Lipoic acid (LA) is synthesized from octanoic acid and cysteine in mitochondria and is present in very small amounts in mammalian tissues as a cofactor of pyruvate dehydrogenase, $\alpha$-keto-glutarate dehydrogenase and branched-chain- $\alpha$-ketoacid dehydrogenase enzymes (19). LA has direct and indirect antioxidant properties and has been proposed as a potent antioxidant for the treatment of DM and its complications $(19,20)$. In our previous study, we showed that LA increased serum PON activities and decreased serum lipid peroxidation in experimental diabetes (18).

Although it has been shown that LA has beneficial effects in myocardial infarction (21) and DM (19), its effect on preventing cardiac damage in myocardial-infarcted diabetic patients is not known. We did not encounter any studies which investigated the 
effect of LA on PON enzyme activities or lipid peroxidation in myocardial infarction.

The aim of our study was to investigate the effect of LA in the prevention of myocardial infarction in diabetic rats. For this purpose, the effect of LA on cardiac damage, serum MDA levels and paraoxonase and lactonase activities were investigated in ISO-induced myocardial infarcted non-diabetic and diabetic rats.

\section{Materials and methods}

\section{Animal experiments}

This study was approved by the Animal Ethics Committee of our University. The experiments were performed using SpragueDawley male rats provided by The Unite of the Laboratory Animals for Experimental Studies. The animal room was maintained at a temperature of $22 \pm 2{ }^{\circ} \mathrm{C}$ and a relative humidity of $55 \pm 15$ $\%$ with a 12 -h light-dark cycle.

In the present study, diabetic myocardial infarction model of Goyal et al (22) was used with some modifications. We choose $40 \mathrm{mg} / \mathrm{kg}$ dose of STZ to induce DM as we used in our previous studies (18).

Thirty-five rats were equally and randomly divided into five groups as control, ISO, LA+ISO, STZ+ISO and STZ+LA+ISO. One rat from STZ+ISO group (5 days after STZ injection) and one rat from STZ+LA+ISO group (12 days after STZ injection) died during the course of experiment. Also one rat from STZ+LA+ISO group was resistant after STZ injection and its tail vein blood glucose level was lower than $300 \mathrm{mg} / \mathrm{dL}$ (22). Experimental procedure was repeated with 3 new rats instead of them. None of the rats was death after ISO injections.

In the beginning of the experiment, fasting blood glucose levels from tail vein were measured in all rats by using AccuChek Active blood glucose test meter (Roche Diagnostics, Basel, Switzerland). To induce diabetes, a single dose of STZ (40 mg/kg, dissolved in $\mathrm{pH} 4.5$ citrate buffer) was injected intraperitoneally to STZ+ISO and STZ+LA+ISO groups. Simultaneously, a single dose of citrate buffer ( $\mathrm{pH} 4.5)$ was injected intraperitoneally to other groups. 72 hours after STZ or citrate buffer injection, fasting blood glucose levels from tail vein were measured in all rats. Rats with fasting blood glucose levels higher than $300 \mathrm{mg} / \mathrm{dL}$ were considered diabetic (22).

Immediately after fasting blood glucose measurement, LA was injected to LA+ISO and STZ+LA+ISO groups. LA was dissolved in sterile $1 \mathrm{M}$ sodium bicarbonate and diluted with sterile saline (18) and was injected intraperitoneally for 14 days $(10 \mathrm{mg} / \mathrm{kg} /$ day) to LA+ISO and STZ+LA+ISO groups (23). To other groups, vehicle was injected instead of LA. $10 \mathrm{mg} / \mathrm{kg} /$ day LA dose used for the treatment of rats was similar to $600 \mathrm{mg} /$ day LA, a dose for diabetic neuropathy treatment $(24,25)$, for $60-70 \mathrm{~kg}$ person, which was also used in previous studies for the treatment of diabetic rats $(18,23)$. We also choose this LA dose by taking into consideration of Al-Rasheed et al (26) who reported that $100 \mathrm{mg} / \mathrm{kg} /$ day LA had adverse cardiac responses in diabetic rats.

Before the ISO injection, blood glucose levels from tail vein were measured in all animals after 12 hour fasting to confirm diabetes. To induce MI, ISO $(85 \mathrm{mg} / \mathrm{kg} /$ day dissolved in $0.9 \%$ $\mathrm{NaCl}$ ) was injected intraperitoneally to ISO, LA+ISO, STZ+ISO and STZ+LA+ISO groups on the days 13 and 14 of LA treatment. Simultaneously, vehicle was injected to control group.

24 hours after the second ISO injection, all rats were sacrificed by collecting blood samples and heart tissues under xylazine $(10 \mathrm{mg} / \mathrm{kg})$ and ketamine hydrochloride $(50 \mathrm{mg} / \mathrm{kg})$ anaesthesia. Blood samples were centrifuged $3000 \mathrm{~g}$ for 10 minutes at $4{ }^{\circ} \mathrm{C}$ and serum was separated. Sera were kept at $-70^{\circ} \mathrm{C}$ for subsequent biochemical analyses.

\section{Histopathological examinations}

The rat hearts were fixed in $10 \%$ formaldehyde for 8 hours. All hearts were divided into two with a sagittal section perpendicular to the interventricular septa and they were sampled showing both ventricle walls and the interventricular septa. The tissues were embedded into paraffin after an overnight alcohol processing. Five micrometer thick sections were taken on slide and stained with routine hematoxylin-eosin stain. Also, sections from each block were taken on coated slides for immunohistochemistry. The hematoxylin-eosin stained slides were evaluated under a light microscope (Olympus BX51) in a blinded manner. The necrosis of the striated muscle and the mixed inflammatory infiltration composed of neutrophil leukocytes and histiocytes were graded in a semi quantitative manner; 0: none, 1: mild, 2: moderate, 3: severe. Immunohistochemically leucocyte common antigen (mouse monoclonal, Neomarkers, USA) was applied to all sections to highlight the infiltrating leucocytes in the heart wall.

\section{Biochemical measurements}

Serum paraoxonase activities were measured according to the method of Gan et al (27) and serum lactonase activities according to the method of Draganov et al (28) Serum was pre-incubated with $5 \times 10^{-6} \mathrm{~mol} / \mathrm{L}$ eserine for 10 minutes at $25^{\circ} \mathrm{C}$ to inhibit butyrylcholinesterase activity. All the measurements were performed at $25^{\circ} \mathrm{C}$. Serum paraoxonase and lactonase activities were determined by measuring the initial rate of substrate hydrolysis at 412 $\mathrm{nm}$ for paraoxonase activity and $270 \mathrm{~nm}$ for lactonase activity in the assay mixture containing $1 \mathrm{mM} \mathrm{CaCl}, 1 \mathrm{mM}$ substrate (respectively; paraoxon and dihydrocoumarin) in $50 \mathrm{mM}$ Tris- $\mathrm{HCl}$ buffer ( $\mathrm{pH}$ 8.0). The blank sample containing incubation mixture without serum was run simultaneously for a correction of spontaneous substrate breakdown. Enzyme activities were calculated from molar extinction coefficients, $17000 \mathrm{M}^{-1} \mathrm{~cm}^{-1}$ for paraoxonase activity (27) and $1295 \mathrm{M}^{-1} \mathrm{~cm}^{-1}$ for lactonase activity (28) and were expressed as $\mathrm{U} / \mathrm{mL}$. One enzyme unit was defined as the amount of enzyme, which hydrolyzes $1 \mathrm{nmol}$ paraoxon for paraoxonase activity and $1 \mu \mathrm{mol}$ dihydrocoumarin for lactonase activity in one minute at $25^{\circ} \mathrm{C}$.

Serum MDA levels were measured for the determination of lipid peroxidation according to the method of Ohkawa et al (29) The results were expressed in nmol MDA $/ \mathrm{mL}$.

Serum alanine aminotransferase (ALT) activities were measured by Advia 1800 clinical chemistry analyzer (Siemens Healthcare Diagnostics GmbH, Erlangen, Germany) using the original kit. 
664-669

Tab. 1. Body weights and blood glucose levels of groups.

\begin{tabular}{|c|c|c|c|c|c|c|}
\hline \multicolumn{2}{|c|}{ Parameters / Groups } & Control & ISO & $\mathrm{LA}+\mathrm{ISO}$ & STZ+ISO & $\mathrm{STZ}+\mathrm{LA}+\mathrm{ISO}$ \\
\hline \multirow{4}{*}{ 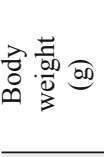 } & İnitial & $287.3 \pm 15.9$ & $284.7 \pm 16.2$ & $282.7 \pm 18.5$ & $279.0 \pm 18.2$ & $277.6 \pm 18.2$ \\
\hline & 72 hours after STZ injection & $284.6 \pm 21.2$ & $282.6 \pm 12.8$ & $281.4 \pm 19.5$ & $240.3 \pm 14.6 *$ & $236.9 \pm 12.9 *$ \\
\hline & Before ISO injection & $288.0 \pm 22.2$ & $285.0 \pm 13.2$ & $284.0 \pm 16.7$ & $204.0 \pm 13.1 *$ & $199.3 \pm 15.0 *$ \\
\hline & Final & $287.9 \pm 21.0$ & $273.3 \pm 14.9$ & $271.0 \pm 15.5$ & $199.7 \pm 12.2 *$ & $191.9 \pm 18.1 *$ \\
\hline \multirow{3}{*}{ 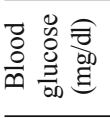 } & İnitial & $106.7 \pm 8.4$ & $106.1 \pm 5.6$ & $101.7 \pm 6.1$ & $110.4 \pm 7.6$ & $107.0 \pm 6.4$ \\
\hline & 72 hours after STZ injection & $112.7 \pm 6.8$ & $105.1 \pm 6.4$ & $107.3 \pm 7.6$ & $379.3 \pm 12.3 *$ & $350.9 \pm 18.9 *$ \\
\hline & Before ISO injection & $104.7 \pm 6.0$ & $106.4 \pm 5.1$ & $102.4 \pm 7.9$ & $445.4 \pm 75.9 *$ & $425.4 \pm 32.8 *$ \\
\hline
\end{tabular}

Results are expressed as mean \pm SD for seven rats in all groups. Data was analyzed by one-way analysis of variance. *: $<<0.05$ compared with control, ISO and LA + ISO groups.

(A)
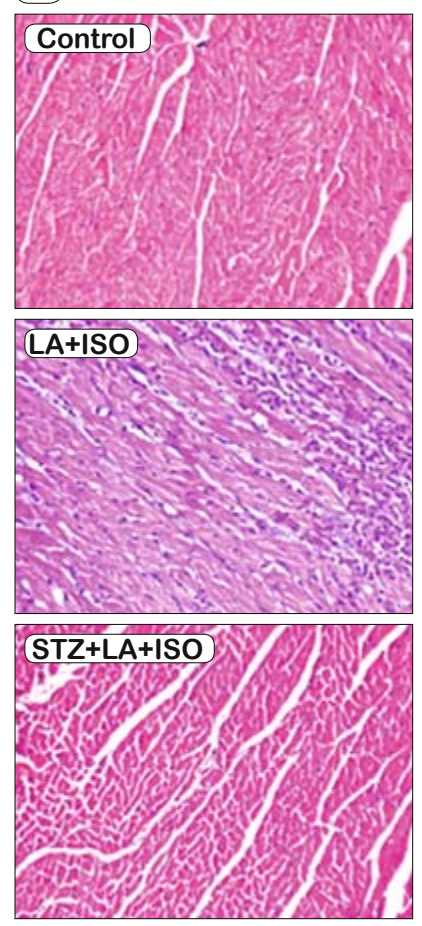

(C)

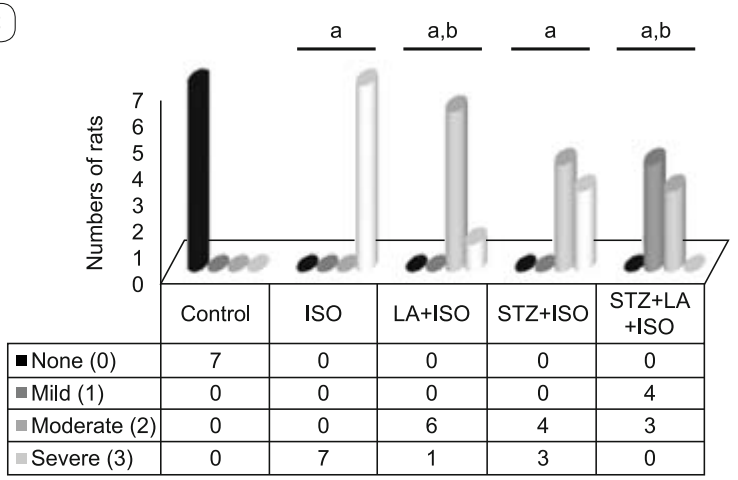

(B)
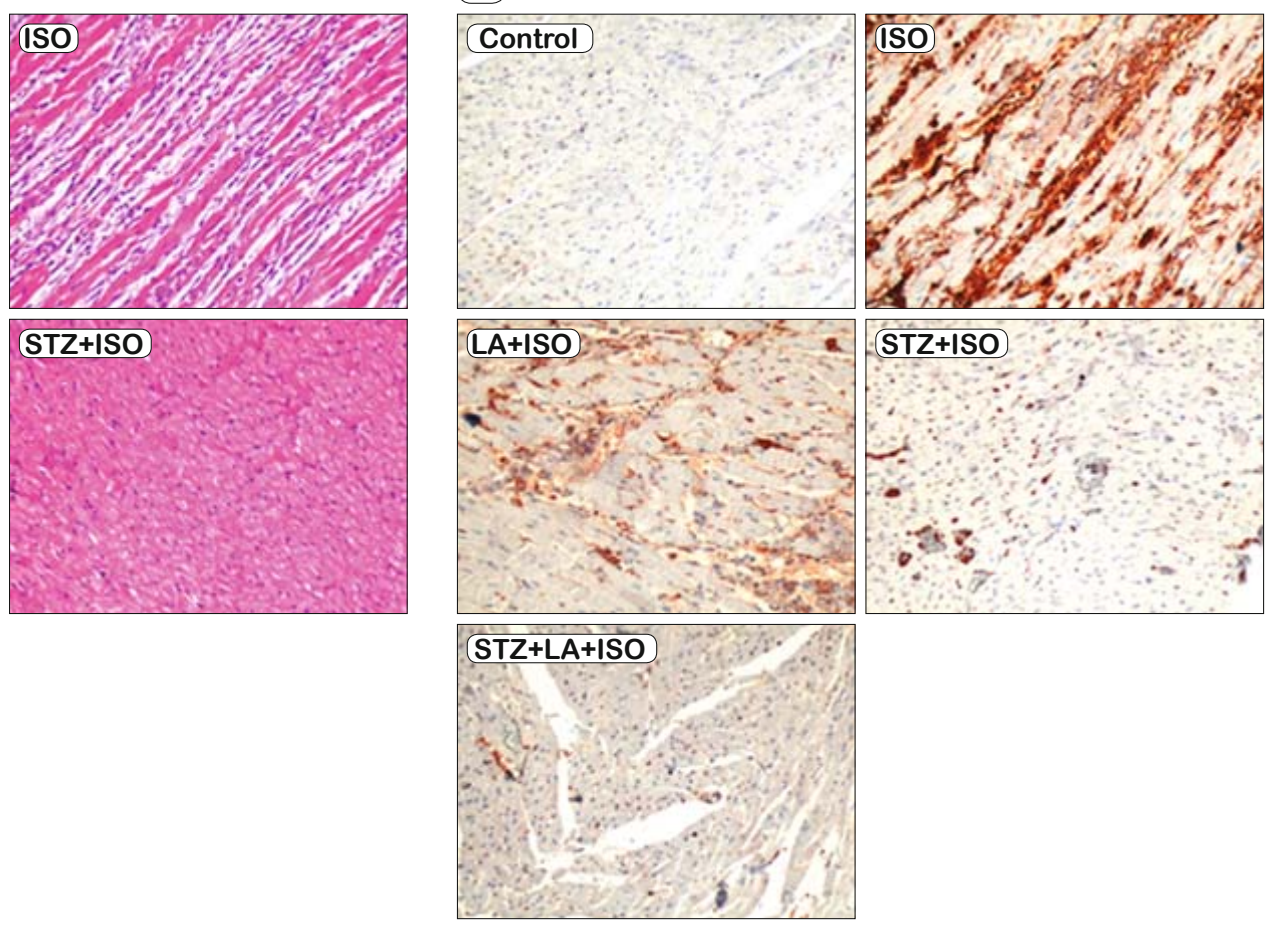

(D)

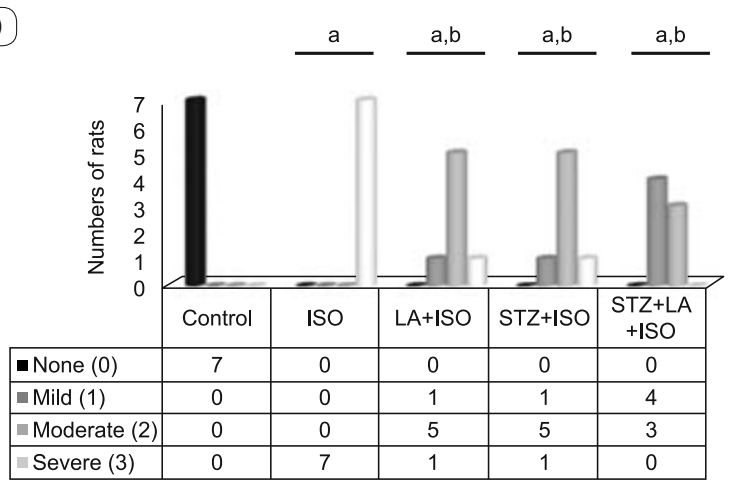

Fig. 1. Histopathological examinations of hearts. A) Slides stained with hematoxylin-eosin (x100), B) Slides stained with leucocyte common antigen immunohistochemically $(\mathbf{x 1 0 0})$, C) Cardiac necrosis scores and D) Leucocyte infiltration scores. Data analyzed by Two-Sample Kolmogorov-Smirnov test. a: $\mathbf{p}<\mathbf{0 . 0 5}$, compared with control group, $b: \mathbf{p}<0.05$, compared with ISO group.

\section{Statistical analysis}

Results were given as the means \pm standard deviation (SD). Non-parametric two-sample Kolmogorov-Smirnov test was used for comparisons of cardiac necrosis and leukocyte infiltration scores of groups. The One-Way ANOVA test was used for comparison of all other parameters among the groups, and then, Tukey or Tamhane post-hoc tests were used for multiple comparisons when the significant difference obtained. SPSS 20.0 (IBM SPSS 
Inc., Chicago, IL, USA) statistical software was used for statistical analysis. $\mathrm{p}<0.05$ was considered as statistical significant.

\section{Results}

Initial weights, 72 hours after the STZ injection, before ISO injection and final body weights of groups are given in the Table 1. There was no significant difference between the initial body weights of groups. 72 hours after the STZ injection, before ISO injection and final body weights in STZ+ISO and STZ+LA+ISO groups were significantly lower when compared to the other groups ( $\mathrm{p}<0.05$ for all).

Initial blood glucose, 72 hours after the STZ injection and before ISO injection blood glucose levels are given in the Table 1. There was no significant difference between the initial blood glucose levels in the groups, 72 hours after the STZ injection and before ISO injection blood glucose levels in STZ+ISO and STZ+LA+ISO groups were significantly higher when compared to the other groups ( $\mathrm{p}<0.05$ for all).

For histopathologic studies, rat hearts were stained with hematoxylin-eosin (Fig. 1a) and leucocyte common antigen immunohistochemically (Fig. 1b). Cardiac necrosis and leucocyte infiltration scores of groups were shown in Fig. 1c and Fig. 1d, respectively. Cardiac necrosis or leucocyte infiltration was not seen in the control group. All of the ISO injected rats have cardiac necrosis and leucocyte infiltration in different grades. Cardiac necrosis scores in ISO injected groups were significantly higher than in the control group ( $p<0.05$ for all). Cardiac necrosis scores in LA+ISO and $\mathrm{STZ}+\mathrm{LA}+\mathrm{ISO}$ groups were significantly lower when compared to the ISO group ( $p<0.05$ for both). Cardiac leucocyte infiltration scores in ISO injected groups were significantly higher than in the control group $(\mathrm{p}<0.05$ for all). Cardiac leucocyte infiltration scores in LA+ISO, STZ+ISO and STZ+LA+ISO groups were significantly lower when compared to the ISO group ( $p<0.05$ for all).

Serum paraoxonase and lactonase activities of the groups are given in Figure 2a and Figure 2b respectively. Serum paraoxonase and lactonase activities in ISO injected groups were significantly lower when compared to the control group $(\mathrm{p}<0.05$ for all). Serum paraoxonase and lactonase activities in LA+ISO group were significantly higher than ISO group $(\mathrm{p}<0.05)$. STZ+ISO and STZ+LA+ISO groups had significantly lower serum paraoxonase and lactonase activities when compared to the LA+ISO group ( $p<0.05$ for both).

Serum MDA levels of groups are given in Figure 2c. Serum MDA levels in ISO, STZ+ISO and STZ+LA+ISO groups were significantly higher when compared to the control group $(\mathrm{p}<0.05$ for all). Serum MDA levels in LA+ISO group were significantly lower when compared to the ISO group $(p<0.05)$. Serum MDA levels in STZ+ISO and STZ+LA+ISO groups were significantly higher than ISO and LA+ISO group ( $p<0.05$ for all).

Serum ALT activities of the groups are given in Figure 2d. Serum ALT activities in STZ+ISO and STZ+LA+ISO groups
(A)

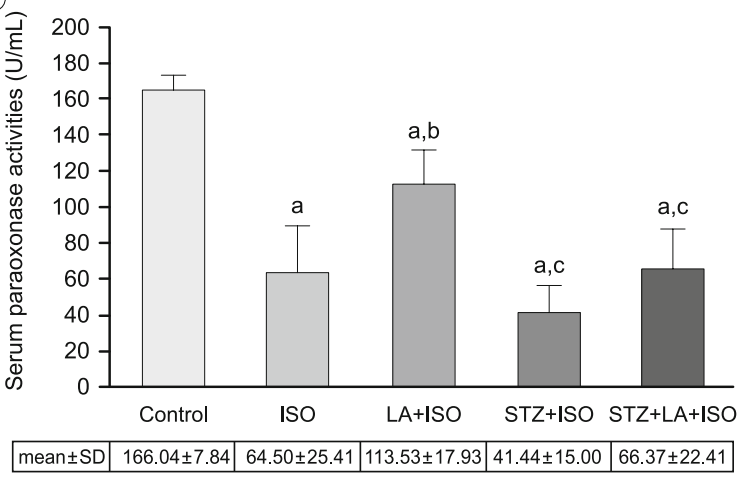

(C)

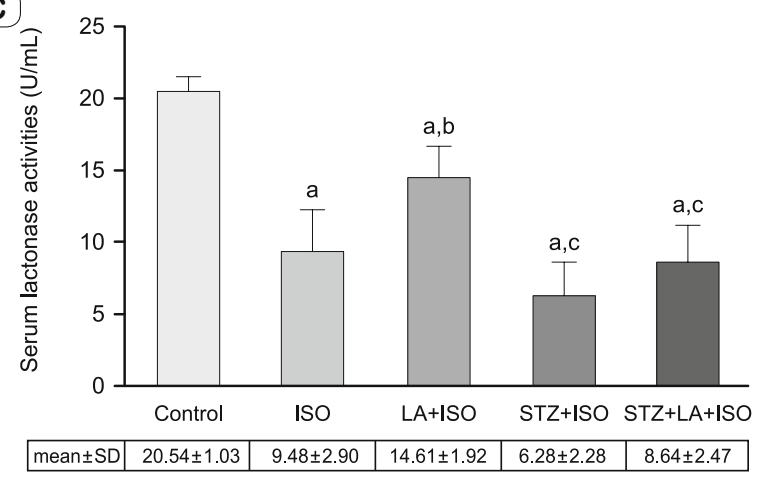

(B)

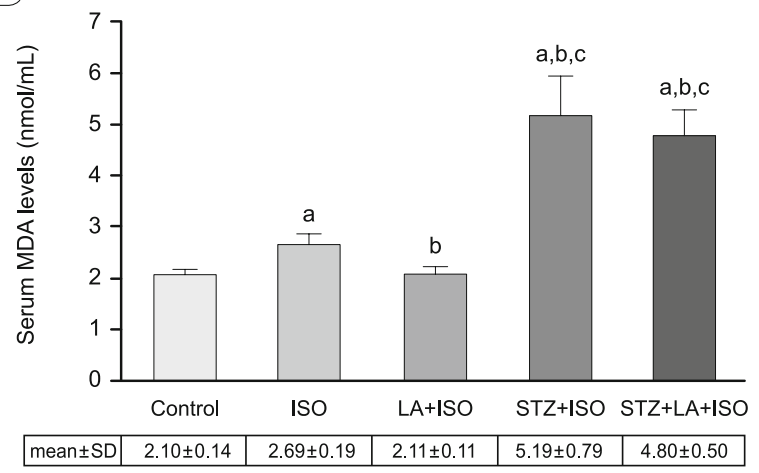

(D)

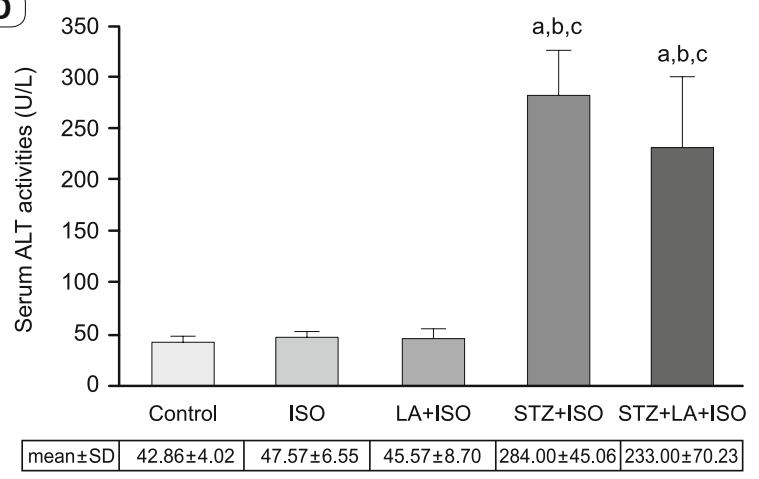

Fig. 2. Biochemical parameters of groups. a) Serum paraoxonase activities, b) Serum lactonase activities, c) Serum MDA levels and d) Serum ALT activities of groups. Results are expressed as the mean \pm SD for seven rats in all groups. Data analyzed by One-Way ANOVA. a: $p<0.05$, compared with control group, $b: \mathbf{p}<\mathbf{0 . 0 5}$, compared with ISO group, $\mathrm{c}: \mathrm{p}<\mathbf{0 . 0 5}$, compared with LA+ISO group. 
were significantly increased as compared to the other groups ( $\mathrm{p}$ $<0.05$ for all).

\section{Discussion}

Increased oxidative stress contributes to the pathogenesis of DM, which is a multifactorial metabolic disorder (30). DM increases the risk of myocardial infarction (8).

LA has gained a considerable attention as an antioxidant therapeutic choice for the use for the treatment of diabetic complications (20). LA acts by multiple mechanisms; it quenches reactive oxygen species, reduces the oxidized forms of other antioxidants, repairs oxidized proteins, regulates gene expression and chelates metal ions (19).

PON1 and PON3, which are primarily synthesized by the liver, are antioxidant and anti-atherogenic enzymes in circulation bound to HDL (13). Although most known activity of PON enzymes is paraoxonase activity, only PON1 has paraoxonase activity and all paraoxonase enzymes are primarily lactonases (14). Both myocardial infarction $(15,16)$ and $\mathrm{DM}(17,18)$ causes a decrease in the activities of these antioxidant enzymes.

Although it has been shown that LA increased serum PON activities and decreased serum lipid peroxidation in DM (18, 31 ), its effect on these enzyme activities and lipid peroxidation in myocardial infarction was not known. It is known that LA has beneficial effects in both myocardial infarction (21) and DM (19) but its effect on preventing cardiac damage in myocardial-infarcted diabetic patients is not known.

In the present study, we aimed to investigate the effect of LA in the prevention of myocardial infarction in diabetic rats.

STZ injection caused hyperglycemia and weight loss, which confirms the experimental diabetic model and supports the previous studies $(13,18)$. Before ISO injection, to confirm the existence of diabetes, we measured blood glucose level of rats once again and found that STZ-injected rats were still hyperglycaemic. LA treatment did not change blood glucose levels and body weights of rats.

ISO-induced myocardial infarction is a common model used for drug investigation and cardiac function (3). In our study, ISO administration caused a cardiac necrosis and leucocyte infiltration, which confirmed the experimental myocardial infarction (4). LA treatment significantly decreased necrosis and inflammation scores in ISO-induced myocardial infarcted non-diabetic rats but did not change cardiac necrosis or leucocyte infiltration scores in ISO-induced myocardial infarcted diabetic rats. Our findings, indicating that LA treatment significantly decreased necrosis and inflammation scores in ISO-induced myocardial infarcted nondiabetic rats, is supported by Abdelbaky et al (21).

Interestingly, we found that ISO-induced myocardial infarcted non-diabetic rats had significantly higher cardiac leucocyte infiltration scores when compared to ISO-induced myocardial infarcted diabetic rats. In the present study, to induce myocardial infarction, ISO was injected at the dose of $85 \mathrm{mg} / \mathrm{kg} /$ day to rats. A significant decrease in body weights in diabetic rats due to STZ injection was taken into consideration. Lower cardiac leucocyte infiltration scores of ISO-induced myocardial infarcted diabetic rats can result from the injection of lower amount of total ISO to these animals.

In the present study, ISO caused a significant increase in lipid peroxidation in rats that supports the previous studies $(5,16)$. LA treatment prevented an increase in serum lipid peroxidation in myocardial infarcted non-diabetic rats but it did not significantly change serum lipid peroxidation in myocardial infarcted diabetic rats. It is known that ISO causes damage to cardiac tissue by increasing oxidative stress (34). Our finding, which indicates that LA decreases lipid peroxidation and cardiac damage in ISO-induced myocardial infarcted non-diabetic rats, points out that LA may prevent cardiac damage by decreasing oxidative stress.

We found that myocardial infarcted diabetic rats had a significant increase in lipid peroxidation when compared with myocardial infarcted non-diabetic rats. It is known that lipid peroxidation is also increased in STZ-induced diabetes $(18,32)$. According to our results, LA was insufficient to fight with oxidative stress and to prevent cardiac damage caused by STZ+ISO injections.

While paraoxonase activity is mainly specific to PON1 enzyme, sources of lactonase activity in serum are both PON1 and PON3 (14). In our previous study (16), we showed that serum paraoxonase and lactonase enzyme activities are decreased in ISO-induced myocardial infarction. In keeping with that study (16), ISO administration caused a significant decrease on paraoxonase and lactonase activities in the present study. LA treatment prevented the decrease of these activities in myocardial infarcted non-diabetic rats. The prevention of a decrease in PON enzyme activities by LA in myocardial infarcted non-diabetic rats may result from the antioxidant effect of LA. It is known that PON enzymes are modulated and inactivated by oxidative stress (13). Antioxidant LA might decrease the degradation PON1 and PON3 enzymes by decreasing oxidative stress. LA may also increase PON1 and PON3 protein synthesis in liver in myocardial infarcted non-diabetic rats. In our previous report (33), we showed that LA increased both PON1 and PON3 protein synthesis in humanderived liver cells.

LA treatment did not significantly change serum PON enzyme activities in myocardial infarcted diabetic rats in this study. LA was insufficient to prevent oxidative stress in myocardial infarcted diabetic rats. Therefore, it might not have prevented the degradation of PON enzymes fighting with oxidative stress. We found an increase in serum ALT activities, which indicated liver damage in myocardial infarcted diabetic rats, which supports previous reports $(34,35)$. Increased liver damage by STZ injection may contribute to a decreased synthesis of liver PON1 and PON3 in myocardial infarcted diabetic rats. LA could not be effective on PON enzyme synthesis because of liver damage. LA also did not change serum ALT activities.

In the present study, ISO caused an increase in cardiac damage and serum lipid peroxidation, whereas a decrease in serum PON enzyme activities. On the other hand, in myocardial infarcted nondiabetic rats, while LA caused a decrease in cardiac damage and serum lipid peroxidation and an increase in serum PON enzyme activities, it did not change these histopathologic or biochemical parameters in myocardial infarcted diabetic rats. 


\section{Conclusion}

We can report that LA, at the dose of $10 \mathrm{mg} / \mathrm{kg}$, is effective to prevent myocardial infarction in non-diabetic rats, but is insufficient in diabetic rats.

\section{References}

1. Reddy K, Khaliq A, Henning RJ. Recent advances in the diagnosis and treatment of acute myocardial infarction. World J Cardiol 2015; 7 (5): 243-276.

2. Madole MB, Bachewar NP, Aiyar CM. Study of oxidants and antioxidants in patients of acute myocardial infarction. Adv Biomed Res 2015; 4: 41.

3. Ou L, Li W, Liu Y et al. Animal models of cardiac disease and stem cell therapy. Open Cardiovasc Med J 2010; 4: 231-239.

4. Rona G. Catecholamine cardiotoxicity. J Mol Cell Cardiol 1985; 17 (4): 291-306.

5. Hussain Shaik A, Rasool SN, Kareem MA et al. Maslinic acid protects against isoproterenol-induced cardiotoxicity in albino Wistar rats. J Med Food 2012; 15 (8): 741-746.

6. Wong ZW, Thanikachalam PV, Ramamurthy S. Molecular understanding of the protective role of natural products on isoproterenol-induced myocardial infarction: A review. Biomed Pharmacother 2017; 94: 1145-1166.

7. Vaidya V, Gangan N, Sheehan J. Impact of cardiovascular complications among patients with Type 2 diabetes mellitus: a systematic review. Expert Rev Pharmacoecon Outcomes Res 2015; 15 (3): 487-497.

8. Lundberg V, Stegmayr B, Asplund K, Eliasson M, Huhtasaari F. Diabetes as a risk factor for myocardial infarction: population and gender perspectives. J Intern Med 1997; 241 (16): 485-492.

9. Di Filippo C, Cuzzocrea S, Rossi F, Marfella R, D'Amico M. Oxidative stress as the leading cause of acute myocardial infarction in diabetics. Cardiovasc Drug Rev 2006; 24 (2): 77-87.

10. Srinivasan K, Ramarao P. Animal models in type 2 diabetes research: an overview. Indian J Med Res 2007; 125 (3): 451-472.

11. Bashan N, Kovsan J, Kachko I, Ovadia H, Rudich A. Positive and negative regulation of insulin signaling by reactive oxygen and nitrogen species. Physiol Rev 2009; 89 (1): 27-71

12. Del Rio D, Stewart AJ, Pellegrini N. A review of recent studies on malondialdehyde as toxic molecule and biological marker of oxidative stress. Nutr Metab Cardiovasc Dis 2005; 15 (4): 316-328.

13. Précourt LP, Amre D, Denis MC et al. The three-gene paraoxonase family: physiologic roles, actions and regulation. Atherosclerosis 2011; 214 (1): 20-36.

14. Draganov DI, Teiber JF, Speelman A, Osawa Y, Sunahara R, La Du BN. Human paraoxonases (PON1, PON2, and PON3) are lactonases with overlapping and distinct substrate specificities. J Lipid Res 2005; 46 (6): 1239-1247.

15. Ayub A, Mackness MI, Arrol S, Mackness B, Patel J, Durrington PN. Serum paraoxonase after myocardial infarction. Arterioscler Thromb Vasc Biol 1999; 19 (2): 330-335.

16. Ozgun E, Sayilan Ozgun G, Usta U, Eskiocak S, Süer Gökmen S. Melatonin effects on serum paraoxonase and lactonase in experimental myocardial infarction. Türk Klinik Biyokimya Derg 2018; 16 (1): 32-41.

17. Nair SP, Shah NC, Taggarsi A, Nayak U. PONI and its association with oxidative stress in type I and type II diabetes mellitus. Diabetes Metab Syndr 2011; 5 (3): 126-129.
18. Ozgun E, Ozgun GS, Gokmen SS et al. Effect of lipoic acid on serum paraoxonase-1 and paraoxonase-3 protein levels and activities in diabetic rats. Exp Clin Endocrinol Diabetes 2016

19. Golbidi S, Badran M, Laher I. Diabetes and alpha lipoic acid. Front Pharmacol 2011; 2: 69.

20. Rochette L, Ghibu S, Muresan A, Vergely C. Alpha-lipoic acid: molecular mechanisms and therapeutic potential in diabetes. Can J Physiol Pharmacol 2015; 93 (12): 1021-1027.

21. Abdelbaky NA, Al-Rasheed N, Al-Rasheed NM, Zaghloul I, Radwan MA. Alpha-lipoic acid and amlodipine ameliorate myocardial infarction induced by isoproterenol in rats. Int J Acad Res 2009; 1 (1): 68-77.

22. Goyal S, Arora S, Bhatt TK, Das P, Sharma A, Kumari S, Arya DS. Modulation of PPAR- $\gamma$ by telmisartan protects the heart against myocardial infarction in experimental diabetes. Chem Biol Interact 2010; 185 (3): 271-280.

23. Maritim AC, Sanders RA, Watkins JB 3rd. Effects of alpha-lipoic acid on biomarkers of oxidative stress in streptozotocin-induced diabetic rats. J Nutr Biochem 2003; 14 (5): 288-294.

24. McIlduff CE, Rutkove SB. Critical appraisal of the use of alpha lipoic acid (thioctic acid) in the treatment of symptomatic diabetic polyneuropathy. Ther Clin Risk Manag 2011; 7: 377-385

25. Mijnhout GS, Alkhalaf A, Kleefstra N, Bilo HJ. Alpha lipoic acid: a new treatment for neuropathic pain in patients with diabetes. Neth J Med 2010; 68 (4): 158-162.

26. Al-Rasheed N, Al-Rasheed NM, Attia HA et al. Adverse cardiac responses to alpha-lipoic acid in a rat-diabetic model: possible mechanisms? J Physiol Biochem 2013; 69 (4): 761-778.

27. Gan KN, Smolen A, Eckerson HW, La Du BN. Purification of human serum paraoxonase/arylesterase. Evidence for one esterase catalyzing both activities. Drug Metab Dispos 1991; 19 (1): 100-106.

28. Draganov DI, Stetson PL, Watson CE, Billecke SS, La Du BN. Rabbit serum paraoxonase 3 (PON3) is a high density lipoprotein-associated lactonase and protects low density lipoprotein against oxidation. J Biol Chem 2000; 275 (43): 33435-33442.

29. Ohkawa H, Ohishi N, Yagi K. Assay for lipid peroxides in animal tissues by thiobarbituric acid reaction. Anal Biochem 1979; 95 (2): 351-358.

30. Giacco F, Brownlee M. Oxidative stress and diabetic complications. Circulat Res 2010; 107 (9): 1058-1070.

31. Gavrovskaia LK, Ryzhova OV, Safonova AF, Aleksandrova IIa, Sapronov NS. Effect of taurine and thioctacide on carbohydrate metabolism and the antioxydant system in rats with experimental diabetes. Eksp Klin Farmakol 2008; 71 (3): 34-35.

32. Wang L, Wu CG, Fang CQ et al. The protective effect of $\alpha$-Lipoic acid on mitochondria in the kidney of diabetic rats. Int J Clin Exp Med 2013; 6 (2): 90-97.

33. Ozgun E, Sayilan Ozgun G, Tabakcioglu K, Suer Gokmen S, Sut N, Eskiocak S. Effect of lipoic acid on paraoxonase-1 and paraoxonase-3 protein levels, mRNA expression and arylesterase activity in liver hepatoma cells. Gen Physiol Biophys 2017; 36 (4): 465-470.

34. Ghanbari E, Nejati V, Khazaei M. Improvement in serum biochemical alterations and oxidative stress of liver and pancreas following use of royal jelly in streptozotocin-induced diabetic rats. Cell J 2016; 18 (3): 362-370.

35. Yanardag R, Ozsoy-Sacan O, Bolkent S, Orak H, Karabulut-Bulan O. Protective effects of metformin treatment on the liver injury of streptozotocindiabetic rats. Hum Exp Toxicol 2005; 24 (3): 129-135.

Received July 2, 2018. Accepted August 24, 2018. 